\title{
A MODULARITY LIFTING THEOREM FOR WEIGHT TWO HILBERT MODULAR FORMS
}

\author{
Toby Gee
}

\begin{abstract}
We prove a modularity lifting theorem for potentially Barostti-Tate representations over totally real fields, generalising recent results of Kisin.
\end{abstract}

\section{Introduction}

In [Kis04] Mark Kisin introduced a number of new techniques for proving modularity lifting theorems, and was able to prove a very general lifting theorem for potentially Barsotti-Tate representations over $\mathbb{Q}$. In [Kis05] this was generalised to the case of $p$-adic representations of the absolute Galois group of a totally real field in which $p$ splits completely. In this note, we further generalise this result to:

Theorem. Let $p>2$, let $F$ be a totally real field in which $p$ is unramified, and let $E$ be a finite extension of $\mathbb{Q}_{p}$ with ring of integers $\mathcal{O}$. Let $\rho: G_{F} \rightarrow \mathrm{GL}_{2}(\mathcal{O})$ be a continuous representation unramified outside of a finite set of primes, with determinant a finite order character times the p-adic cyclotomic character. Suppose that

(1) $\rho$ is potentially Barsotti-Tate at each $v \mid p$.

(2) $\bar{\rho}$ is modular.

(3) $\left.\bar{\rho}\right|_{F\left(\zeta_{p}\right)}$ is absolutely irreducible.

Then $\rho$ is modular.

We emphasise that the techniques we use are entirely those of Kisin. Our only new contributions are some minor technical improvements; specifically, we are able to prove a more general connectedness result than Kisin for certain local deformation rings, and we replace an appeal to a result of Raynaud by a computation with Breuil modules with descent data.

The motivation for studying this problem was the work reported on in [Gee06], where we apply the main theorem of this paper to the conjectures of [BDJ05]. In these applications it is crucial to have a lifting theorem valid for $F$ in which $p$ is unramified, rather than just totally split.

\section{Connected components}

Firstly, we recall some definitions and theorems from [Kis04]. We make no attempt to put these results in context, and the interested reader should consult section 1 of [Kis04] for a more balanced perspective on this material.

Received by the editors January 26, 2006.

2000 Mathematics Subject Classification. 11F33. 
Let $p>2$ be prime. Let $k$ be a finite extension of $\mathbb{F}_{p}$ of cardinality $q=p^{r}$, and let $W=W(k), K_{0}=W(k)[1 / p]$. Let $K$ be a totally ramified extension of $K_{0}$ of degree $e$. We let $\mathfrak{S}=W[[u]]$, equipped with a Frobenius map $\phi$ given by $u \mapsto u^{p}$, and the natural Frobenius on $W$. Fix an algebraic closure $\bar{K}$ of $K$, and fix a uniformiser $\pi$ of $K$. Let $E(u)$ denote the minimal polynomial of $\pi$ over $K_{0}$.

Let '(Mod / S ) denote the category of $\mathfrak{S}$-modules $\mathfrak{M}$ equipped with a $\phi$-semilinear map $\phi: \mathfrak{M} \rightarrow \mathfrak{M}$ such that the cokernel of $\phi^{*}(\mathfrak{M}) \rightarrow \mathfrak{M}$ is killed by $E(u)$. For any $\mathbb{Z}_{p}$-algebra $A$, set $\mathfrak{S}_{A}=\mathfrak{S} \otimes_{\mathbb{Z}_{p}} A$. Denote by ${ }^{\prime}(\operatorname{Mod} / \mathfrak{S})_{A}$ the category of pairs $(\mathfrak{M}, \iota)$ where $\mathfrak{M}$ is in ${ }^{\prime}(\operatorname{Mod} / \mathfrak{S})$ and $\iota: A \rightarrow \operatorname{End}(\mathfrak{M})$ is a map of $\mathbb{Z}_{p}$-algebras.

We let $(\operatorname{Mod} F I / \mathfrak{S})_{A}$ denote the full subcategory of ' $(\operatorname{Mod} / \mathfrak{S})_{A}$ consisting of objects $\mathfrak{M}$ such that $\mathfrak{M}$ is a projective $\mathfrak{S}_{A}$-module of finite rank.

Choose elements $\pi_{n} \in \bar{K}(n \geq 0)$ so that $\pi_{0}=\pi$ and $\pi_{n+1}^{p}=\pi_{n}$. Let $K_{\infty}=$ $\bigcup_{n \geq 1} K\left(\pi_{n}\right)$. Let $\mathcal{O}_{\mathcal{E}}$ be the $p$-adic completion of $\mathfrak{S}[1 / u]$. Let $\operatorname{Rep}_{\mathbb{Z}_{p}}\left(G_{K_{\infty}}\right)$ denote the category of continuous representations of $G_{K_{\infty}}$ on finite $\mathbb{Z}_{p}$-algebras. Let $\Phi \mathrm{M}_{\mathcal{O}_{\mathcal{E}}}$ denote the category of finite $\mathcal{O}$-modules $M$ equipped with a $\phi$-semilinear map $M \rightarrow M$ such that the induced map $\phi^{*} M \rightarrow M$ is an isomorphism. Then there is a functor

$$
T: \Phi \mathrm{M}_{\mathcal{O}_{\mathcal{E}}} \rightarrow \operatorname{Rep}_{Z_{p}}\left(G_{K_{\infty}}\right)
$$

which is in fact an equivalence of abelian categories (see section 1.1 .12 of [Kis04]). Let $A$ be a finite $\mathbb{Z}_{p}$-algebra, and let $\operatorname{Rep}_{A}^{\prime}\left(G_{K_{\infty}}\right)$ denote the category of continuous representations of $G_{K_{\infty}}$ on finite $A$-modules, and let $\operatorname{Rep}_{A}\left(G_{K_{\infty}}\right)$ denote the full subcategory of objects which are free as $A$-modules. Let $\Phi \mathrm{M}_{\mathcal{O}_{\mathcal{E}}, A}$ denote the category whose objects are objects of $\Phi \mathrm{M}_{\mathcal{O}_{\mathcal{E}}}$ equipped with an action of $A$.

Lemma 2.1. The functor $T$ above induces an equivalence of abelian categories

$$
T_{A}: \Phi \mathrm{M}_{\mathcal{O}_{\mathcal{E}}, A} \rightarrow \operatorname{Rep}_{A}^{\prime}\left(G_{K_{\infty}}\right) .
$$

The functor $T_{A}$ induces a functor

$$
T_{\mathfrak{S}, A}:(\operatorname{Mod} \mathrm{FI} / \mathfrak{S})_{A} \rightarrow \operatorname{Rep}_{A}\left(G_{K_{\infty}}\right) ; \mathfrak{M} \mapsto T_{A}\left(\mathcal{O}_{\mathcal{E}} \otimes_{\mathfrak{S}} \mathfrak{M}\right)
$$

Proof. Lemmas 1.2.7 and 1.2.9 of [Kis04].

Fix $\mathbb{F}$ a finite extension of $\mathbb{F}_{p}$, and a continuous representation of $G_{K}$ on a 2dimensional $\mathbb{F}$-vector space $V_{\mathbb{F}}$. We suppose that $V_{\mathbb{F}}$ is the generic fibre of a finite flat group scheme, and let $M_{\mathbb{F}}$ denote the preimage of $V_{\mathbb{F}}(-1)$ under the equivalence $T_{\mathbb{F}}$ of Lemma 2.1.

In fact, from now on we assume that the action of $G_{K}$ on $V_{\mathbb{F}}$ is trivial, that $k \subset \mathbb{F}$, and that $k \neq \mathbb{F}_{p}$. In applications we will reduce to this case by base change.

Recall from Corollary 2.1.13 of [Kis04] that we have a projective scheme $\mathcal{G} \mathcal{R}_{V_{\mathbb{F}}, 0}$, such that for any finite extension $\mathbb{F}^{\prime}$ of $\mathbb{F}$, the set of isomorphism classes of finite flat models of $V_{\mathbb{F}^{\prime}}=V_{\mathbb{F}} \otimes_{\mathbb{F}} \mathbb{F}^{\prime}$ is in natural bijection with $\mathcal{G} \mathcal{R}_{V_{\mathbb{F}}, 0}\left(\mathbb{F}^{\prime}\right)$. We work below with the closed subscheme $\mathcal{G R}_{V_{\mathbb{F}}, 0}^{\mathbf{v}}$ of $\mathcal{G} \mathcal{R}_{V_{\mathbb{F}}, 0}$, defined in Lemma 2.4.3 of [Kis04], which parameterises isomorphism classes of finite flat models of $V_{\mathbb{F}^{\prime}}$ with cyclotomic determinant.

As in section 2.4.4 of [Kis04], if $\mathbb{F}^{\text {sep }}$ is the residue field of $K_{0}^{\text {sep }}$, and $\sigma \in \operatorname{Gal}\left(K_{0} / \mathbb{Q}_{p}\right)$, we denote by $\epsilon_{\sigma} \in k \otimes_{\mathbb{F}_{p}} \mathbb{F}^{\prime}$ the idempotent which is 1 on the kernel of the map $1 \otimes \sigma: k \otimes_{\mathbb{F}_{p}} \mathbb{F}^{\prime} \rightarrow \mathbb{F}^{\text {sep }}$ corresponding to $\sigma$, and 0 on the other maximal ideals of $k \otimes_{\mathbb{F}_{p}} \mathbb{F}^{\prime}$. 
Lemma 2.2. If $\mathbb{F}^{\prime}$ is a finite extension of $\mathbb{F}$, then the elements of $\mathcal{G} \mathcal{R}_{V_{\mathbb{F}}, 0}^{\mathrm{v}}\left(\mathbb{F}^{\prime}\right)$ naturally correspond to free $k \otimes_{\mathbb{F}_{p}} \mathbb{F}^{\prime}[[u]]$-submodules $\mathcal{M}_{\mathbb{F}^{\prime}} \subset M_{\mathbb{F}^{\prime}}:=M_{\mathbb{F}} \otimes_{\mathbb{F}} \mathbb{F}^{\prime}$ of rank 2 such that:

(1) $\mathcal{M}_{\mathbb{F}^{\prime}}$ is $\phi$-stable.

(2) For some (so any) choice of $k \otimes_{\mathbb{F}_{p}} \mathbb{F}^{\prime}[[u]]$-basis for $\mathcal{M}_{\mathbb{F}^{\prime}}$, for each $\sigma$ the map

$$
\phi: \epsilon_{\sigma} \mathcal{M}_{\mathbb{F}^{\prime}} \rightarrow \epsilon_{\sigma \circ \phi^{-1}} \mathcal{M}_{\mathbb{F}^{\prime}}
$$

has determinant $\alpha u^{e}, \alpha \in \mathbb{F}^{\prime}[[u]]^{\times}$.

Proof. This follows just as in the proofs of Lemma 2.5.1 and Proposition 2.2.5 of [Kis04]. More precisely, the method of the proof of Proposition 2.2.5 of [Kis04] allows one to "decompose" the determinant condition into the condition that for each $\sigma$ we have

$$
\operatorname{dim}_{\mathbb{F}^{\prime}}\left(\epsilon_{\sigma \circ \phi^{-1}} \mathcal{M}_{\mathbb{F}^{\prime}} / \phi\left(\epsilon_{\sigma} \mathcal{M}_{\mathbb{F}^{\prime}}\right)\right)=e,
$$

and then an identical argument to that in the proof of Lemma 2.5.1 [Kis04] shows that this condition is equivalent to the stated one.

We now number the elements of $\operatorname{Gal}\left(K_{0} / \mathbb{Q}_{p}\right)$ as $\sigma_{1}, \ldots, \sigma_{r}$, in such a way that $\sigma_{i+1}=\sigma_{i} \circ \phi^{-1}$ (where we identify $\sigma_{r+1}$ with $\left.\sigma_{1}\right)$. For any sublattice $\mathfrak{M}_{\mathbb{F}}$ in $(M o d / \mathfrak{S})_{\mathbb{F}}$ and any $\left(A_{1}, \ldots, A_{r}\right) \in \mathcal{M}_{2}(\mathbb{F}((u)))^{r}$, we write $\mathfrak{M}_{\mathbb{F}} \sim A$ if there exist bases $\left\{\mathbf{e}_{1}^{i}, \mathbf{e}_{2}^{i}\right\}$ for $\epsilon_{\sigma_{i}} \mathcal{M}_{\mathbb{F}}$ such that

$$
\phi\left(\begin{array}{l}
\mathbf{e}_{1}^{i} \\
\mathbf{e}_{2}^{i}
\end{array}\right)=A_{i}\left(\begin{array}{l}
\mathbf{e}_{1}^{i+1} \\
\mathbf{e}_{2}^{i+1}
\end{array}\right) .
$$

If we have fixed such a choice of basis, then for any $\left(B_{1}, \ldots, B_{r}\right) \in \mathrm{GL}_{2}\left(k_{r}((u))\right)^{r}$ we denote by $B \mathfrak{M}$ the module generated by $\left\langle B_{i}\left(\begin{array}{c}\mathbf{e}_{1}^{i} \\ \mathbf{e}_{2}^{i}\end{array}\right)\right\rangle$, and consider $B \mathfrak{M}$ with respect to the basis given by these entries.

Proposition 2.3. Let $\mathbb{F}^{\prime} / \mathbb{F}$ be a finite extension. Suppose that $x_{1}, x_{2} \in \mathcal{G R}_{V_{\mathbb{F}}, 0}^{\mathrm{v}}\left(\mathbb{F}^{\prime}\right)$ and that the corresponding objects of $(\operatorname{Mod} / \S)_{\mathbb{F}^{\prime}}, \mathfrak{M}_{\mathbb{F}^{\prime}, 1}$ and $\mathfrak{M}_{\mathbb{F}^{\prime}, 2}$ are both nonordinary. Then (the images of) $x_{1}$ and $x_{2}$ both lie on the same connected component of $\mathcal{G} \mathcal{R}_{V_{\mathbb{F}}, 0}^{\mathrm{v}}\left(\mathbb{F}^{\prime}\right)$.

Proof. Replacing $V_{\mathbb{F}}$ by $\mathbb{F}^{\prime} \otimes_{\mathbb{F}} v_{\mathbb{F}}$, we may assume that $\mathbb{F}^{\prime}=\mathbb{F}$. Suppose that $\mathfrak{M}_{\mathbb{F}, 1} \sim A$. Then $\mathfrak{M}_{\mathbb{F}, 2}=B \cdot \mathfrak{M}_{\mathbb{F}, 1}$ for some $B \in \mathrm{GL}_{2}\left(k_{r}((u))\right)^{r}$, and $\mathfrak{M}_{\mathbb{F}, 2} \sim\left(\phi\left(B_{i}\right) \cdot A_{i} \cdot B_{i+1}^{-1}\right)$. Each $B_{i}$ is uniquely determined up to left multiplication by elements of $\mathrm{GL}_{2}(\mathbb{F}[[u]])$, so by the Iwasawa decomposition we may assume that each $B_{i}$ is upper triangular. By Lemma $2.2, \operatorname{det} \phi\left(B_{i}\right) \operatorname{det} B_{i+1}^{-1} \in \mathbb{F}[[u]]^{\times}$for all $i$, which implies that $\operatorname{det}\left(B_{i}\right) \in \mathbb{F}[[u]]^{\times}$ for all $i$, so that the diagonal elements of $B_{i}$ are $\mu_{1}^{i} u^{-a_{i}}, \mu_{2}^{i} u^{a_{i}}$ for $\mu_{1}^{i}, \mu_{2}^{i} \in \mathbb{F}[[u]]^{\times}$, $a_{i} \in \mathbb{Z}$. Replacing $B_{i}$ with $\operatorname{diag}\left(\mu_{1}^{i}, \mu_{2}^{i}\right)^{-1} B_{i}$, we may assume that $B_{i}$ has diagonal entries $u^{-a_{i}}$ and $u^{a_{i}}$.

We now show that $x_{1}$ and $x_{2}$ are connected by a chain of rational curves, using the following lemma:

Lemma 2.4. Suppose that $\left(N_{i}\right)$ are nilpotent elements of $M_{2}(\mathbb{F}((u)))$ such that $\mathfrak{M}_{\mathbb{F}, 2}=(1+N) \cdot \mathfrak{M}_{\mathbb{F}, 1}$. If $\phi\left(N_{i}\right) A N_{i+1}^{\text {ad }} \in M_{2}(F[[u]])$ for all $i$, then there is a map $\mathbb{P}^{1} \rightarrow \mathcal{G R}_{V_{\mathbb{F}}, 0}^{\mathrm{v}}$ sending 0 to $x_{1}$ and 1 to $x_{2}$.

Proof. Exactly as in the proof of Lemma 2.5.7 of [Kis04]. 
In fact, we will only apply this lemma in situations where all but one of the $N_{i}$ are zero, so that the condition of the lemma is automatically satisfied.

Lemma 2.5. With respect to some basis, $\phi: M_{\mathbb{F}} \rightarrow M_{\mathbb{F}}$ is given by $\left(\begin{array}{ll}1 & 0 \\ 0 & 1\end{array}\right)$.

Proof. This is immediate from the definition of $M_{\mathbb{F}}$ (recall that we have assumed that the action of $G_{K}$ on $V_{\mathbb{F}}$ is trivial).

Let $v_{1}, v_{2}$ be a basis as in the lemma, and let $\mathfrak{M}_{\mathbb{F}}$ be the sub- $k \otimes_{\mathbb{F}_{p}} \mathbb{F}[[u]]$-module generated by $u^{e /(p-1)} v_{1}$ and $v_{2}$ (note that the assumption that the action of $G_{K}$ on $V_{\mathbb{F}}$ is trivial guarantees that $e \mid(p-1))$. Then $\mathfrak{M}_{\mathbb{F}}$ corresponds to an object of $\mathcal{G R}_{V_{\mathbb{F}}, 0}^{\mathbf{v}}\left(\mathbb{F}^{\prime}\right)$, and $\mathfrak{M}_{\mathbb{F}} \sim\left(A_{i}\right)$ where each $A_{i}=\left(\begin{array}{cc}u^{e} & 0 \\ 0 & 1\end{array}\right)$, so that $\mathfrak{M}_{\mathbb{F}}$ is ordinary.

Furthermore, every object of $\mathcal{G} \mathcal{R}_{V_{\mathbb{F}}, 0}^{\mathbf{v}}\left(\mathbb{F}^{\prime}\right)$ is given by $B \cdot \mathfrak{M}_{\mathbb{F}}$ for some $B=\left(B_{i}\right)$, where $B_{i}=\left(\begin{array}{cc}u^{-a_{i}} & v_{i} \\ 0 & u^{a_{i}}\end{array}\right)$, and $\phi\left(B_{i}\right) A_{i} B_{i+1}^{-1} \in M_{2}(\mathbb{F}[[u]])$ for all $i$. Examining the diagonal entries of $\phi\left(B_{i}\right) A_{i} B_{i+1}^{-1}$, we see that we must have $e \geq p a_{i}-a_{i+1} \geq 0$ for all $i$.

Lemma 2.6. We have $e /(p-1) \geq a_{i} \geq 0$ for all $i$. Furthermore, if any $a_{i}=0$ then all $a_{i}=0 ;$ and if any $a_{i}=e /(p-1)$, then all $a_{i}=e /(p-1)$.

Proof. Suppose that $a_{j} \leq 0$. Then $p a_{j} \geq a_{j+1}$, so $a_{j+1} \leq 0$. Thus $a_{i} \leq 0$ for all $i$. But adding the inequalities gives $(p-1)\left(a_{1}+\cdots+a_{r}\right) \geq 0$, so in fact $a_{1}=\cdots=a_{r}=0$. The other half of the lemma follows in a similar fashion.

Note that the ordinary objects are precisely those with all $a_{i}=0$ or all $a_{i}=$ $e /(p-1)$. We now show that there is a chain of rational curves linking any nonordinary point to the point corresponding to $C \cdot \mathfrak{M}_{\mathbb{F}}$, where $C_{i}=\left(\begin{array}{cc}u^{-1} & 0 \\ 0 & u\end{array}\right)$.

Choose a non-ordinary point $D \cdot \mathfrak{M}_{\mathbb{F}}, D_{i}=\left(\begin{array}{cc}u^{-b_{i}} & w_{i} \\ 0 & u^{b_{i}}\end{array}\right)$. We claim that there is a chain of rational curves linking this to the point $D^{\prime} \cdot \mathfrak{M}_{\mathbb{F}}, D_{i}^{\prime}=\left(\begin{array}{cc}u^{-b_{i}} & 0 \\ 0 & u^{b_{i}}\end{array}\right)$. Clearly, it suffices to demonstrate that there is a rational curve from $D \cdot \mathfrak{M}_{\mathbb{F}}$ to the point $D^{j} \cdot \mathfrak{M}_{\mathbb{F}}$, where

$$
D_{i}^{j}= \begin{cases}D_{i}, & i \neq j \\
\left(\begin{array}{cc}
u^{-b_{j}} & 0 \\
0 & u^{b_{j}}
\end{array}\right), & i=j .\end{cases}
$$

But this is easy; just apply Lemma 2.4 with $N=\left(N_{i}\right)$,

$$
N_{i}= \begin{cases}0, & i \neq j \\
\left(\begin{array}{l}
0-w_{j} u^{-b_{j}} \\
0
\end{array}\right), & i=j .\end{cases}
$$

It now suffices to show that there is a chain of rational curves linking $D^{\prime} \cdot \mathfrak{M}_{\mathbb{F}}$ to $C \cdot \mathfrak{M}_{\mathbb{F}}$. Suppose that $D^{\prime \prime} \cdot \mathfrak{M}_{\mathbb{F}}$ also corresponds to a point of $\mathcal{G} \mathcal{R}_{V_{\mathbb{F}}, 0}^{\mathbf{v}}(\mathbb{F})$, where for some $j$ we have

$$
D_{i}^{\prime \prime}= \begin{cases}D_{i}^{\prime}, & i \neq j \\
\left(\begin{array}{cc}
u^{1-b_{j}} & 0 \\
0 & u^{b_{j}-1}
\end{array}\right), & i=j .\end{cases}
$$

Then we claim that there is a rational curve linking $D^{\prime} \cdot \mathfrak{M}_{\mathbb{F}}$ and $D^{\prime \prime} \cdot \mathfrak{M}_{\mathbb{F}}$. Note that $D^{\prime \prime}=E D^{\prime}$, where

$$
E_{i}= \begin{cases}1, & i \neq j \\
\left(\begin{array}{cc}
u^{-1} & 0 \\
0 & u
\end{array}\right), & i=j .\end{cases}
$$


Since $\left(\begin{array}{cc}u^{-1} & 0 \\ 0 & u\end{array}\right)=\left(\begin{array}{cc}0 & 1 \\ -1 & 2 u\end{array}\right)\left(\begin{array}{cc}2 & -u \\ u^{-1} & 0\end{array}\right)$, and $\left(\begin{array}{cc}0 & 1 \\ -1 & 2 u\end{array}\right) \in \mathrm{GL}_{2}(\mathbb{F}[[u]])$, we can apply Lemma 2.4 with

$$
N_{i}= \begin{cases}0, & i \neq j \\
\left(\begin{array}{cc}
1 & -u \\
u^{-1}-1
\end{array}\right), & i=j .\end{cases}
$$

Proposition 2.3 now follows from:

Lemma 2.7. If $e /(p-1)>a_{i}>0$ and $e \geq p a_{i}-a_{i+1} \geq 0$ for all $i$, and not all the $a_{i}$ are equal to 1 , then for some $j$ we can define

$$
a_{i}^{\prime}= \begin{cases}a_{i}, & i \neq j \\ a_{j}-1, & i=j\end{cases}
$$

and we have $e \geq p a_{i}^{\prime}-a_{i+1}^{\prime} \geq 0$ for all $i$.

Proof. Suppose not. Then for each $i$, either $p a_{i-1}-\left(a_{i}-1\right)>e$, or $p\left(a_{i}-1\right)-a_{i+1}<0$; that is, either $p a_{i-1}-a_{i}=e$, or $p-1 \geq p a_{i}-a_{i+1} \geq 0$. Comparing the statements for $i, i+1$, we see that either $p a_{i}-a_{i+1}=e$ for all $i$, or $p-1 \geq p a_{i}-a_{i+1} \geq 0$ for all $i$. In the former case we have $a_{i}=e /(p-1)$ for all $i$, a contradiction. In the latter case, summing the inequalities gives $r(p-1) \geq(p-1)\left(a_{1}+\cdots+a_{r}\right) \geq(r+1)(p-1)$, a contradiction.

\section{Modularity lifting theorems}

The results of section 2 can easily be combined with the machinery of [Kis04] to yield modularity lifting theorems. For example, we have the following:

Theorem 3.1. Let $p>2$, let $F$ be a totally real field, and let $E$ be a finite extension of $\mathbb{Q}_{p}$ with ring of integers $\mathcal{O}$. Let $\rho: G_{F} \rightarrow \mathrm{GL}_{2}(\mathcal{O})$ be a continuous representation unramified outside of a finite set of primes, with determinant a finite order character times the p-adic cyclotomic character. Suppose that

(1) $\rho$ is potentially Barsotti-Tate at each $v \mid p$.

(2) There exists a Hilbert modular form $f$ of parallel weight 2 over $F$ such that $\bar{\rho}_{f} \sim \bar{\rho}$, and for each $v \mid p, \rho$ is potentially ordinary at $v$ if and only if $\rho_{f}$ is.

(3) $\left.\bar{\rho}\right|_{F\left(\zeta_{p}\right)}$ is absolutely irreducible, and if $p>3$ then $\left[F\left(\zeta_{p}\right): F\right]>3$.

Then $\rho$ is modular.

Proof. The proof of this theorem is almost identical to the proof of Theorem 3.5.5 of [Kis04]. Indeed, the only changes needed are to replace property (iii) of the field $F^{\prime}$ chosen there by "(iii) If $v \mid p$ then $\bar{\rho} \mid G_{F_{v}}$ is trivial, and the residue field at $v$ is not $\mathbb{F}_{p}$ ", and to note that Theorem 3.4.11 of [Kis04] is still valid in the context in which we need it, by Proposition 2.3.

For the applications to $\bmod p$ Hilbert modular forms in [Gee06] it is important not to have to assume that $\rho$ is potentially ordinary at $v$ if and only if $\rho_{f}$ is. Fortunately, in [Gee06] it is only necessary to work with totally real fields in which $p$ is unramified, and in that case we are able, following [Kis05], to remove this assumption. 
Theorem 3.2. Let $p>2$, let $F$ be a totally real field in which $p$ is unramified, and let $E$ be a finite extension of $\mathbb{Q}_{p}$ with ring of integers $\mathcal{O}$. Let $\rho: G_{F} \rightarrow \mathrm{GL}_{2}(\mathcal{O})$ be a continuous representation unramified outside of a finite set of primes, with determinant a finite order character times the p-adic cyclotomic character. Suppose that

(1) $\rho$ is potentially Barsotti-Tate at each $v \mid p$.

(2) $\bar{\rho}$ is modular.

(3) $\left.\bar{\rho}\right|_{F\left(\zeta_{p}\right)}$ is absolutely irreducible.

Then $\rho$ is modular.

Proof. Firstly, note that by a standard result (see e.g. [BDJ05]) we have $\bar{\rho} \sim \bar{\rho}_{f}$, where $f$ is a form of parallel weight 2. We now follow the proof of Corollary 2.13 of [Kis05]. Let $\mathcal{S}^{\prime}$ denote the set of $v \mid p$ such that $\left.\rho\right|_{G_{v}}$ is potentially ordinary. After applying Lemma 3.3 below, we may assume that $\bar{\rho} \sim \bar{\rho}_{f}$, where $f$ is a form of parallel weight 2 , and $\rho_{f}$ is potentially ordinary and potentially Barsotti-Tate for all $v \in \mathcal{S}^{\prime}$.

We may now make a solvable base change so that the hypotheses on $F$ in Theorem 3.1 are still satisfied, and in addition $[F: \mathbb{Q}]$ is even, and at every place $v \mid p f$ is either unramified or special of conductor 1. By our choice of $f,\left.\rho_{f}\right|_{G_{v}}$ is Barsotti-Tate and ordinary at each place $v \in \mathcal{S}^{\prime}$. In order to apply Theorem 3.1, we need to check that we can replace $f$ by a form $f^{\prime}$ such that $\bar{\rho} \sim \bar{\rho}_{f^{\prime}}$, and $\rho_{f^{\prime}}$ is Barsotti-Tate at all $v \mid p$ and is ordinary if and only if $\rho$ is. That is, we wish to choose $f^{\prime}$ so that $\rho_{f^{\prime}}$ is Barsotti-Tate and ordinary at all places $v \in \mathcal{S}^{\prime}$, and is Barsotti-Tate and non-ordinary at all other places dividing $p$. The existence of such an $f^{\prime}$ follows at once from the proof of Theorem 3.5.7 of [Kis04]. The theorem then follows from Theorem 3.1.

Lemma 3.3. Let $F$ be a totally real field in which $p$ is unramified, and $\mathcal{S}^{\prime}$ a set of places of $F$ dividing $p$. Let $f$ be a Hilbert modular cusp form over $F$ of parallel weight 2 , with $\bar{\rho}_{f}$ absolutely irreducible, and suppose that for $\left.v \in \mathcal{S}^{\prime} \bar{\rho}_{f}\right|_{G_{F}}$ is the reduction of a potentially Barsotti-Tate representation of $G_{F_{v}}$ which is also potentially ordinary.

Then there is a Hilbert modular cusp form $f^{\prime}$ over $F$ of parallel weight 2 with $\bar{\rho}_{f^{\prime}} \sim \bar{\rho}_{f}$, and such that for all $v \in \mathcal{S}^{\prime}, \rho_{f^{\prime}}$ becomes ordinary and Barsotti-Tate over some finite extension of $F_{v}$.

Proof. We follow the proof of Lemma 2.14 of [Kis05], indicating only the modifications that need to be made. Replacing the appeal to [CDT99] with one to Proposition 1.1 of [Dia05], the proof of Lemma 2.14 of [Kis05] shows that we can find $f^{\prime}$ such that $\bar{\rho}_{f^{\prime}} \sim \bar{\rho}_{f}$, and such that for all $v \in \mathcal{S}^{\prime}, \rho_{f^{\prime}}$ becomes Barsotti-Tate over $F_{v}\left(\zeta_{q_{v}}\right)$, where $q_{v}$ is the degree of the residue field of $F$ at $v$. Furthermore, we can assume that the type of $\left.\rho_{f^{\prime}}\right|_{G_{F v}}$ is $\widetilde{\omega}_{1} \oplus \widetilde{\omega}_{2}$, where $\left.\bar{\rho}_{f^{\prime}}\right|_{G_{F_{v}}} \sim\left(\begin{array}{cc}\omega_{1} \chi & * \\ 0 & \omega_{2}\end{array}\right)$, where $\chi$ is the cyclotomic character, and a tilde denotes the Teichmuller lift. Let $\mathcal{G}$ denote the $p$-divisible group over $\mathcal{O}_{F_{v}\left(\zeta_{q_{v}}\right)}$ corresponding to $\left.\rho_{f^{\prime}}\right|_{F_{v}\left(\zeta_{q_{v}}\right)}$ Then by a scheme-theoretic closure argument, $\mathcal{G}[p]$ fits into a short exact sequence

$$
0 \rightarrow \mathcal{G}_{1} \rightarrow \mathcal{G}[p] \rightarrow \mathcal{G}_{2} \rightarrow 0 .
$$

The information about the type then determines the descent data on the Breuil modules corresponding to $\mathcal{G}_{1}$ and $\mathcal{G}_{2}$. We will be done if we can show that $\mathcal{G}_{1}$ is multiplicative and $\mathcal{G}_{2}$ is étale. However, by the hypothesis on $\mathcal{S}^{\prime}$ we can write down a multiplicative group scheme $\mathcal{G}_{1}^{\prime}$ with the same descent data and generic fibre as $\mathcal{G}_{1}$. 
Then Lemma 3.4 below shows that $\mathcal{G}_{1}$ is indeed multiplicative. The same argument shows that $\mathcal{G}_{2}$ is étale.

Lemma 3.4. Let $k$ be a finite field of characteristic $p$, and let $L=W(k)[1 / p]$. Fix $\pi=(-p)^{1 /\left(p^{d}-1\right)}$ where $d=\left[k: \mathbb{F}_{p}\right]$, and let $K=L(\pi)$. Let $E$ be a finite field containing $k$. Let $\mathcal{G}$ and $\mathcal{G}^{\prime}$ be finite flat rank one E-module schemes over $\mathcal{O}_{K}$ with generic fibre descent data to L. Suppose that the generic fibres of $\mathcal{G}$ and $\mathcal{G}^{\prime}$ are isomorphic as $G_{L}$-representations, and that $\mathcal{G}$ and $\mathcal{G}^{\prime}$ have the same descent data. Then $\mathcal{G}$ and $\mathcal{G}^{\prime}$ are isomorphic.

Proof. This follows from a direct computation using Breuil modules with descent data. Specifically, it follows at once from Example A.3.3 of [Sav06], which computes the generic fibre of any finite flat rank one $E$-module scheme over $\mathcal{O}_{K}$ with generic fibre descent data to $L$.

\section{References}

[BDJ05] K. Buzzard, F. Diamond, and F. Jarvis, On Serre's conjecture for mod l Galois representations over totally real fields, in preparation, 2005.

[CDT99] B. Conrad, F. Diamond, and R. Taylor, Modularity of certain potentially Barsotti-Tate Galois representations, J. Amer. Math. Soc. 12 (1999), no. 2, 521-567.

[Dia05] F. Diamond, A correspondence between representations of local Galois groups and Lie-type groups, to appear in L-functions and Galois representations (Durham 2004), 2005.

[Gee06] Toby Gee, On the weights of mod $p$ Hilbert modular forms. Preprint 2006.

[Kis04] Mark Kisin, Moduli of finite flat group schemes, and modularity. Preprint 2004.

[Kis05] - Modularity for some geometric Galois representations, to appear in L-functions and Galois representations (Durham 2004), 2005.

[Sav06] David Savitt, Breuil modules for Raynaud schemes, appendix to [Gee06], 2006.

Department of Mathematics, Imperial College London, London SW7 2AZ, United KingDOM

E-mail address: toby.gee@ic.ac.uk 